\title{
Hypertriglyceridaemia as a cause of acute pancreatitis
}

\section{Hipertriglicerydemia jako przyczyna ostrego zapalenia trzustki}

\author{
Monika A. Kozłowska-Geller ${ }^{1}$, Jacek Bicki²,3 \\ 1Department of Physiology, Pathophysiology and Clinical Immunology, Collegium Medicum, Jan Kochanowski University, Kielce, Poland \\ Head of Collegium Medicum: Prof. Marianna Janion MD, PhD \\ ${ }^{2}$ Collegium Medicum, Jan Kochanowski University, Kielce, Poland \\ Head of the Collegium: Prof. Marianna Janion MD, PhD \\ ${ }^{3}$ Department of Clinic General Oncological and Endocrinological Surgery, Regional Hospital, Kielce, Poland \\ Head of the Department: Prof. Stanisław Głuszek MD, PhD
}

Medical Studies/Studia Medyczne 2020; 36 (1): 63-65

DOI: https://doi.org/10.5114/ms.2020.94092

Acute pancreatitis is an inflammatory disease of the pancreas associated with varying degrees of autodigestion, oedema, necrosis, and haemorrhage of the pancreatic tissue. Hypertriglyceridaemia is considered to be the third most common cause of acute pancreatitis, and a serum level > $1000 \mathrm{mg} / \mathrm{dl}$ has been strongly associated with acute pancreatitis.

A 39-year-old man with body mass index (BMI) $28 \mathrm{~kg} / \mathrm{m}^{2}$, without a past medical history, was admitted to hospital in good general condition, with epigastric pain, nausea and vomiting for 2 days, after alcohol drinking. His physical examination was notable for guarding and tenderness in the epigastric region - on the Trapnell scale $-2^{\text {nd }}$ degree, arterial pressure within normal limits, regular heart rate-98/minute. His initial diagnostics workup is summarised in Table 1 - noteworthy is the significantly elevated triglyceride level $>8000$ with inability to calculate low-density lipoprotein (LDL) cholesterol fraction.

Abdominal computed tomography showed diffuse inflammatory stranding around the pancreas concerning the pancreatitis with no evidence of gallstones or biliary sludge. He denied any history of gallstones, pancreatitis, new medications, or recent abdominal procedures. Hospitalisation was complicated by exudate in both pleural cavities with the need for their drainage. He was treated with high-volume intravenous fluids, intravenous insulin regimen, heparin drip, pain control, lipid-lowering therapy with a statin and fibrate, carbapenem antibiotics, and nutritional treatment. The patient's pain was well controlled, triglyceride levels decreased gradually, and the patient was discharged in stable condition with his home medications along with the addition of long-term statin and fibrate therapy and therapy to reduce insulin resistance.

Hypertriglyceridaemia is the third leading cause of acute pancreatitis after gallstones and alcohol abuse. The incidence of acute pancreatitis in patients with hypertriglyceridaemia is estimated at $5-77 \%$ by various authors. The pancreas plays an important role in lipid homeostasis, although the pancreas prefers lip- ids as a metabolic substrate. Acute pancreatitis produced experimentally in animals results regularly in the transient elevation of serum lipids for 1 to 4 days. However, investigations of others authors indicate that abnormalities in lipid metabolism are constant [1].

It is still unknown if hypertriglyceridaemia is the cause or consequence of acute pancreatitis. It has been suggested that the production of acute pancreatitis in hyperlipaemia is a result of the embolisation of pancreatic vessels by fat.

One of the most attractive suggestions explaining the relationship between increased triglyceride levels and acute pancreatitis is the influence of pancreatic lipase on triglycerides [2].

The pathogenesis of elevated triglycerides causing acute pancreatitis involves the breakdown of triglyceride into toxic free fatty acids (FFA) by the pancreatic lipases, which is the actual underlying cause of lipo-

Table 1. Initial laboratory work up

\begin{tabular}{|lc|}
\hline Parameter & Results \\
WBC & $8.9 \mu / \mathrm{l}$ \\
Haemoglobin & $20.5 \mathrm{~g} / \mathrm{dl}$ \\
Haematocrit & $44.4 \%$ \\
CRP & 3.48 \\
Sodium & $117 \mathrm{mEq} / \mathrm{l}$ \\
Potassium & $4.0 \mathrm{mEq} / \mathrm{l}$ \\
Serum creatinine & Unable to calculate \\
Glucose & $339 \mathrm{mg} / \mathrm{dl}$ \\
Aspartate aminotransferase & $209 \mathrm{IU} / \mathrm{l}$ \\
Alanine aminotransferase & $169 \mathrm{IU} / \mathrm{l}$ \\
Total bilirubin & $0.74 \mathrm{mg} / \mathrm{dl}$ \\
Lipase & $549 \mathrm{IU} / \mathrm{l}$ \\
Triglyceride & $8092 \mathrm{mg} / \mathrm{dl}$ \\
LDL & Unable to calculate \\
\hline
\end{tabular}


toxicity during acute pancreatitis. The severity of the damage is twofold because there is an inflammatory response caused by pancreatitis as well as the injury caused by lipotoxicity from triglyceride hydrolysis [3].

We assume that not only dietary lipids and carbohydrates but also free fatty acids secreted from adipocytes could be involved in pancreatic $\beta$-cell dysfunction. Such phenomena are well known as lipotoxicity. In addition, it is known that decreased insulin effect leads to the reduction of lipoprotein lipase activity. Because activated lipoprotein lipase induces metabolisms of triglyceride-rich lipoprotein, decreased insulin effect leads to the reduction of lipoprotein lipase activity and the elevation of triglyceride-rich lipoprotein.

The high concentrations of triglycerides in the human pancreas might be hydrolysed by pancreatic lipase, of which a relatively high activity is present in pancreatic capillaries, resulting in the local release of large quantities of fatty acids. The source of triglycerides can be adipose tissue around and within the pancreas, as well as plasma triglycerides. Pancreatic lipase is specific for fatty acid residue at positions sn- 1 and sn-3 of glycerol moiety, and hydrolysis of triglycerides by this enzyme gives mainly free saturated fatty acids with some amount of di- and monoglycerides. Studies using an isolated pancreatic acini model suggested that damage to acinar cells is caused by the cytotoxic effect of fatty acids. Also, diglycerides cause structural and functional disturbances in cellular membrane. Free fatty acids released during the lipolysis of triglycerides are generally believed to be bound to serum albumin and in this form are not toxic. If large quantities of fatty acids were released from triglycerides, they would be toxic to the tissues and could result in acinar cell and/or capillary injury $[1,4]$.

The results of this experiment supported mainly the role of albumin in the binding of fatty acids and the prevention of their cytotoxic effect [1].

The toxicity of FFA is debated in terms of the activation of trypsinogen by acidosis due to the presence of FFA, the direct effects of fatty acids or their salts on the cell membrane, and disturbed micro-circulation caused by the damaging action of FFA on the endothelium.

It has been suggested that increased serum triglycerides may predispose a patient to develop acute pancreatitis if exposed to other provoking factors, such as alcohol or gallstones. Experimental data showed that hyperlipaemia intensifies the course of both acute oedematous and acute necrotising pancreatitis in rats. It is possible that subtle abnormalities in the catabolism of chylomicron remnants might represent a central pathogenic event as a coadjuvant in acute pancreatitis associated with such factors as gallstone disease or alcohol consumption.

One of theory assumes the influence of alcohol, one of the aetiological factors of acute pancreatitis, on the level of triglycerides. The production of triglycerides increases in the presence of alcohol. In animals under the influence of ethanol the synthesis of lipoproteins was unaltered, while fractional catabolic rates were significantly reduced. Thus, ethanol-induced changes in hepatic metabolism are responsible for delayed lipoprotein clearance.

Whether the actual episode of pancreatitis is caused directly by the alcohol or by the lipid abnormality is unclear $[1,4,5]$.

Excessive alcohol intake increases secretion of gastric and pancreatic juices. Simultaneous papillary oedema and spasm of the sphincter of Oddi develop. As a result, the pressure of the pancreatic duct elevates and gives rise to a rupture of the small pancreatic duct and to the development of acute pancreatitis. Other theories also exist, as an obstructive hypersecretion with formation of a protein plug and the metabolic influence of alcohol and its metabolites $[1,6]$.

Serum and urinary amylase activity are frequently normal in hyperlipaemic patients with acute pancreatitis. The mechanism is not understood. Hypertriglyceridaemia interferes with amylase measurement. Fallat et al. suggested that plasma triglycerides either suppress or inhibit serum amylase activity.

The risk of acute pancreatitis associated with BMI is partly mediated through higher triglycerides. The most common causes of hypertriglyceridaemia-induced acute pancreatitis include lipid disorders, alcohol abuse, medication adverse effects, or poorly controlled diabetes mellitus [7].

There is a limitation in this case report. The patient's genetic background and laboratory data for primary hyperlipidaemia such as familial hyperlipidaemia were not clear. In general, in order to perform its definite diagnosis, it is necessary to examine several points such as the apolipoprotein activity, phenotype of apolipoprotein and lipoprotein lipase activity. First, he had no family history of hyperlipidaemia. Second, there were no findings in physical examination such as xanthelasma palpebrarum, tuberous xanthoma, and plane xanthoma.

Therefore, although we think that very severe hypertriglyceridaemia in this subject was induced by markedly disturbed lifestyle and poorly controlled abnormal glycaemia, we cannot exclude the possibility that he had a primary hyperlipidaemia such as familial hyperlipidaemia.

Taken together, we should bear in mind that disturbed lifestyle and abnormal glycaemia lead to very severe hypertriglyceridaemia. Total care for abnormal glycaemia and hypertriglyceridaemia is very important for the improvement of very severe hypertriglyceridaemia.

Very severe hypertriglyceridaemia is classified as levels above $2000 \mathrm{mg} / \mathrm{dl}$. Management includes targeting pancreatitis with intravenous fluids, pain con- 
trol, and nutritional support [8]. While apheresis with therapeutic plasma exchange is a known option for severe hypertriglyceridaemia, we present a case with management with intravenous fluids, subcutaneous insulin, statins, and fibrates in a patient with a triglyceride level above $8000 \mathrm{mg} / \mathrm{dl}$, who presented with severe epigastric pain radiating to her back.

The standard of care in patients with hypertriglyceridaemia-induced acute pancreatitis with worrisome features including but not limited to hypocalcaemia, elevated temperature, and elevated white blood cells count is plasmapheresis, and if not feasible or available, then intravenous insulin is to be administered. One study showed that octreotide, a somatostatin analogue, was only able to inhibit the release of one pancreatic enzyme - amylase - persistently, showing no effects in levels of trypsin or chymotrypsin secretions [9].

Despite the positive outcome in this case, our patient's hospital course was complicated by bibasilar pleural effusions, multiple abdominal fluid collections, cyclical fevers, and continued abdominal pain. It can be safely assumed that our more conservative approach to the management of this case played a role in the protracted hospital course for this patient, and adherence to the accepted standard of care including plasmapheresis would have led to a shorter and less complicated hospital course as well as a reduction of the overall cost of treatment for this patient.

\section{Conflict of interest}

The authors declare no conflict of interest.

\section{References}

1. Panek J. Hipertriglycerydemia and acute pancreatitis. Chirurgia Pol 2001; 3: 97-104.

2. Kalra SS, Innabi A, Runnstrom M, Lascano J, Patel DC. Severe hypertriglyceridemia, acute pancreatitis and chyloptysis. QJM 2020; 113: 47-48.

3. Eriksson M, Angelin B. How to handle hypertriglyceridaemia in acute pancreatitis - still a vote for conservatives? J Intern Med 2019; 286: 723-725.

4. Sahu KK, Mishra AK, Lal A, Silverman ES. Update on management of hypertriglyceridaemia-induced acute pancreatitis. BMJ Case Rep 2019; 12: pii: e231703.

5. Jo SI, Chang JH, Kim TH, Kim CW, Kim JK, Han SW. Subsets associated with developing acute pancreatitis in patients with severe hypertriglyceridemia and the severity of pancreatitis. Pancreatology 2019; 19: 795-800.

6. Kozieł D, Kozłowska M, Deneka J, Matykiewicz J, Głuszek S. Retrospective analysis of clinical problems concerning acute pancreatitis in one treatment center. Gastroenterology Rev 2013; 8: 320-326.

7. Bajaj B, Grandhe S, Duong H, Ratnayake SN. A rare case of acute pancreatitis due to very severe hypertriglyceridemia treated with subcutaneous insulin and lipid lowering drugs. AME Case Rep 2019; 3: 26.

8. Irie S, Anno T, Kawasaki F, Shigemoto R, Nakanishi S, Ka$\mathrm{ku} \mathrm{K}$, Kaneto H. Severe hypertriglyceridemia in a subject with disturbed life style and poor glycemic control without recurrence of acute pancreatitis: a case report. BMC Endocr Disord 2019; 19: 92.

9. Shemesh E, Zafrir B. hypertriglyceridemia-related pancreatitis in patients with type 2 diabetes: links and risks. Diabetes Metab Syndr Obes 2019; 12: 2041-2052.

\section{Address for correspondence}

Monika A. Kozłowska-Geller MD, PhD

Department of Physiology, Pathophysiology and Clinical Immunology

Collegium Medicum

Jan Kochanowski University

al. IX Wieków Kielc 19a, 25-317 Kielce, Poland

Phone: +48 41602390428

E-mail: monika.kozlowska.chir@onet.pl 